\title{
Dosimetric impact of source-positioning uncertainty in high-dose-rate balloon brachytherapy of breast cancer
}

\author{
Yongbok Kim, PhD \\ Department of Radiation Oncology, The University of Arizona, Tucson, AZ, USA
}

\begin{abstract}
Purpose: To evaluate the dosimetric impact of source-positioning uncertainty in high-dose-rate (HDR) balloon brachytherapy of breast cancer.

Material and methods: For 49 HDR balloon patients, each dwell position of catheter(s) was manually shifted distally (+) and proximally (-) with a magnitude from 1 to $4 \mathrm{~mm}$. Total 392 plans were retrospectively generated and compared to corresponding clinical plans using 7 dosimetric parameters: dose $\left(\mathrm{D}_{95}\right)$ to $95 \%$ of planning target volume for evaluation (PTV_EVAL), and volume covered by $100 \%$ and $90 \%$ of the prescribed dose (PD) ( $\mathrm{V}_{100}$ and $\left.\mathrm{V}_{90}\right)$; skin and rib maximum point dose $\left(D_{\max }\right)$; normal breast tissue volume receiving $150 \%$ and $200 \%$ of $P D\left(V_{150}\right.$ and $\left.V_{200}\right)$.

Results: PTV_EVAL dosimetry deteriorated with larger average/maximum reduction (from $\pm 1 \mathrm{~mm}$ to $\pm 4 \mathrm{~mm}$ ) for larger source position uncertainty ( $p$ value < 0.0001): from $1.0 \% / 2.5 \%, 3.3 \% / 5.9 \%, 6.3 \% / 10.0 \%$ to $9.8 \% / 14.5 \%$ for $\mathrm{D}_{95}$; from $1.0 \% / 2.6 \%, 3.1 \% / 5.7 \%, 5.8 \% / 8.9 \%$ to $8.7 \% / 12.3 \%$ for $\mathrm{V}_{100}$; from $0.2 \% / 1.5 \%, 1.0 \% / 4.0 \%, 2.7 \% / 6.8 \%$ to $5.1 \% / 10.3 \%$ for $V_{90} . \geq \pm 3 \mathrm{~mm}$ shift reduced average $\mathrm{D}_{95}$ to $<95 \%$ and average $\mathrm{V}_{100}$ to $<90 \%$. While skin and rib $\mathrm{D}_{\max }$ change was case-specific, its absolute change $(\mid \Delta$ (Value) $\mid)$ showed that larger shift and high dose group had larger variation compared to smaller and lower dose group ( $p$ value $<0.0001)$, respectively. Normal breast tissue $V_{150}$ variation was case-specific and small. Average $\left|\Delta\left(\mathrm{V}_{150}\right)\right|$ was $0.2 \mathrm{cc}$ for the largest shift $( \pm 4 \mathrm{~mm})$ with maximum $<1.7 \mathrm{cc}$. $\mathrm{V}_{200}$ was increased with higher elevation for larger shift: from $6.4 \mathrm{cc} / 9.8 \mathrm{cc}, 7.0 \mathrm{cc} / 10.1 \mathrm{cc}, 8.0 \mathrm{cc} / 11.3 \mathrm{cc}$ to $9.2 \mathrm{cc} /$ $13.0 \mathrm{cc}$.

Conclusions: The tolerance of $\pm 2 \mathrm{~mm}$ recommended by AAPM TG 56 is clinically acceptable in most clinical cases. However, special attention should be paid to a case where both skin and rib are located proximally to balloon, and the orientation of balloon catheter(s) is vertical to these critical structures. In this case, sufficient dosimetric planning margins are required.
\end{abstract}

Key words: balloon, breast cancer, HDR brachytherapy, positioning uncertainty.

\section{Purpose}

In the three-dimension (3D) computed tomography (CT) image-based treatment planning and delivery using the ${ }^{192}$ Ir high-dose-rate (HDR) afterloader, there are several factors associated with source-positioning uncertainty. The first is the reconstruction uncertainty of 3D planning $\mathrm{CT}$ images in the commercial treatment planning system (TPS). The second results from the nature of manual process in catheter-positioning during 3D CT image-based planning. Particularly, the catheter(s) inside the balloon can be easily identified in the CT image based planning for HDR balloon breast implant. Because image contrast fluid is injected into the balloon, the catheter(s) can be shown either as clearly as a black line due to its low density or as a white line if a dummy metal wire (high density) is inserted through the catheter(s). However, the blurring artifact at the tip of catheter makes it inaccurate to define the tip of catheter on the planning CT images. This makes all possible dwell positions along the catheter have the same magnitude of positioning uncertainty because they all are determined by the single coordinate of catheter tip. And the last is the mechanical accuracy of the afterloader in positioning the ${ }^{192} \mathrm{Ir}$ source to the intended dwell positions for radiation delivery. The afterloader positioning accuracy within $\pm 1 \mathrm{~mm}$ has been considered as clinically acceptable [1]. In the American Association of Physicists in Medicine (AAPM) task group 56 (TG 56) [2], the clinically acceptable accuracy in positioning an ${ }^{192}$ Ir source was recommended within $\pm 2 \mathrm{~mm}$ relative to the applicator system in HDR brachytherapy.

However, it is not well known how much these source-positioning uncertainties perturb the delivered dose compared to the planned dose. Therefore, it is clin- 
ically important to investigate the overall dosimetric impact from these uncertainties combined together. In conventional 2D image-based planning, a point dose is used for prescription and dosimetric reporting. On the contrary, in the 3D CT image-based planning, the 3D CT images enable to compute dose-to-volume information such as dose volume histogram (DVH) for plan evaluation. Therefore, the source-positioning uncertainty can be easily translated into its clinical impact, i.e., DVH change.

In the literature, the same investigators published two Monte Carlo simulation studies [3, 4] regarding dose perturbation due to source positioning uncertainty in HDR balloon brachytherapy of breast cancer. One study [3] was performed for a single MammoSite patient whose skin spacing was $0.7 \mathrm{~cm}$. The other study [4] was performed for a phantom with three different sizes of the balloon $(4,5$, and $6 \mathrm{~cm}$ diameter). In both studies, an HDR ${ }^{192}$ Ir source was positioned at the center of balloon (a single dwell position method) to generate the reference treatment plan. Both studies reported the same amount of dose perturbation due to positioning uncertainty: $+1 \mathrm{~mm}$ and $+2 \mathrm{~mm}$ positioning uncertainty reduced the surface dose at $1 \mathrm{~cm}$ away from the balloon by $7 \%$ and $14 \%$ of the prescribed dose, respectively, while increasing dose at other part of target volume by $9 \%$ and $19 \%$, respectively. In the second study [4], the maximum dose perturbation was observed in the smallest diameter of balloon. Therefore, both studies suggested that a maximum source deviation should be $\leq 1 \mathrm{~mm}$ for clinically acceptable positioning uncertainty.

However, there are several concerns to apply the suggestion from these studies to clinical cases. In the clinical treatment planning, it has been known that multiple dwell position method together with surface optimization can produce better dose distribution than a single dwell position method $[5,6,7,8]$. Dose perturbation due to source positioning uncertainty is highly dependent upon the reference dose distribution. In these two studies $[4,5]$, the reference dose distribution was less clinically relevant because a single dwell position method was used. Second, in order to provide clinically useful guidelines, the sample size should be large enough to include various clinical cases in terms of different balloon positions relative to organs at risk (OARs). In addition, in 3D CT image-based treatment planning, clinically relevant dosimetric parameter is not only target coverage but also OARs doses for plan evaluation. Therefore, in this study source positioning uncertainty was simulated for 49 clinical HDR balloon breast patients and its dosimetric impact was investigated for target coverage and OARs dose.

\section{Material and methods \\ HDR balloon patients}

A single lumen MammoSite ${ }^{\circledR}$ applicator (Hologic Inc., Bedford, MA, USA) was implanted for 25 patients and Contura ${ }^{\circledR}$ multi-lumen balloon (MLB) applicator (Hologic Inc., Bedford, MA, USA) was implanted for 24 patients. For those 49 HDR balloon implants, the average \pm standard deviation (minimum/maximum) value of the volume of planning target volume for evaluation (PTV_EVAL) was 93.4 cc \pm 20.3 cc (59.3-143.7.0 cc).

\section{D CT image-based treatment planning}

The total dose of $34 \mathrm{~Gy}$ was prescribed to the $1 \mathrm{~cm}$ surface expanding from the intracavitary balloon and delivered twice a day with at least 6 hours apart on five consecutive working days using an HDR ${ }^{192}$ Ir source. A 3D CT image based treatment plan was made by following a national joint clinical trial by the National Surgical Adjuvant Breast and Bowel Project/the Radiation Therapy Oncology Group (NSABP B-39/RTOG 0413) [9]. The thickness of CT slice was $3 \mathrm{~mm}$ for single lumen MammoSite ${ }^{\circledR}$ (Hologic Inc.) applicator and $2 \mathrm{~mm}$ for Contura ${ }^{\circledR}$ MLB (Hologic Inc.) applicator, respectively.

First, a volume of spherical shell was constructed by excluding balloon volume from the volume of $1 \mathrm{~cm}$ expansion of balloon in 3D. Afterwards, breast tissue underlying $0.5 \mathrm{~cm}$ close to skin surface, and chest wall and pectoralis muscles are excluded from the spherical shell if the balloon is located close to the skin and rib. The final volume is considered as the PTV_EVAL. Normal breast tissue, skin, and rib were delineated on 3D CT images as OARs and their DVHs were generated accordingly. In order to objectively report the maximal point dose of skin, a virtual skin volume was constructed as $0.5 \mathrm{~cm}$ expansion from the skin surface outside the patient body. Hence, the maximal point dose of this virtual skin volume is located on the skin surface the same as the manual method to report maximal point dose of the skin [10].

For the possible multiple dwell positions defined by the catheters inside the balloon, volume optimization technique commercially available in a TPS (BrachyVision $^{\mathrm{TM}}$ version 8.1.2.0, Varian Medical Systems Inc., Palo Alto, CA, USA) was utilized to determine a set of optimal dwell time distribution. In this TPS, AAPM TG 43 formalism was used for dose calculation without taking tissue heterogeneity into account. The planning goals are adapted from the NSABP B-39/RTOG 0413 protocol [9] and Contura ${ }^{\circledR}$ registry protocol [11]. For the target coverage, dose $\left(D_{95}\right)$ to the $95 \%$ of the volume of PTV_EVAL is preferred to be more than $95 \%$ of the prescribed dose (PTV_EVAL $D_{95} \geq 95 \%$ ). If it is difficult, at least $90 \%$ of PTV_EVAL volume is covered with $90 \%$ of the prescribed dose $\left(\mathrm{V}_{90} \geq 90 \%\right)$ after subtracting air gap and seroma volume relative to the PTV_EVAL volume. The dose to OARs was limited as follow. The maximal point dose $\left(D_{\max }\right)$ to skin and rib was limited to $125 \%$ and $145 \%$ of the prescribed dose, respectively (skin $D_{\max } \leq 125 \%$ and rib $D_{\max } \leq 145 \%$ ). To avoid necrosis of breast tissue, the volume of high dose region within the normal breast tissue was limited, so that the volume $\left(\mathrm{V}_{150}\right.$ and $\left.\mathrm{V}_{200}\right)$ receiving $150 \%$ and $200 \%$ of the prescribed dose can be less than $50 \mathrm{cc}$ and $10 \mathrm{cc}$, respectively $\left(\mathrm{V}_{150} \leq 50 \mathrm{cc}\right.$ and $\mathrm{V}_{200}$ $\leq 10 \mathrm{cc}$ ). For a clinically difficult case, in which the balloon is located close to both skin and rib, either target coverage or dose to OARs was compromised to produce a clinically acceptable plan. 


\section{Source-positioning uncertainty simulation}

In this study, it is assumed that all source-positioning uncertainty in the 3D CT image-based treatment planning and delivery could be combined together and considered as the source-positioning uncertainty ranging from $\pm 1 \mathrm{~mm}$ to $\pm 4 \mathrm{~mm}$ although AAPM TG 56 guideline is $\pm 2 \mathrm{~mm}$ tolerance and afterloader positioning accuracy is $\pm 1 \mathrm{~mm}$. The positioning uncertainty ranging from $\pm 1 \mathrm{~mm}$ to $\pm 4 \mathrm{~mm}$ was simulated to investigate its dosimetric impact for 49 clinical CT image based HDR planning data.

For 25 single lumen MammoSite ${ }^{\circledR}$ balloon HDR patients, all source dwell positions were manually shifted along the catheter distally $(+)$ and proximally $(-)$ with a magnitude of $1 \mathrm{~mm}, 2 \mathrm{~mm}, 3 \mathrm{~mm}$, and $4 \mathrm{~mm}$. A 3D visual inspection was performed to verify the shift of source positions relative to the balloon. For 24 Contura ${ }^{\circledR}$ MLB HDR patients, the same source position shift simulation as for single lumen MammoSite ${ }^{\circledR}$ patients was performed to all five catheters simultaneously. Although in a real clinical case, the source positioning uncertainty occurs individually for each catheter, it is impractical to simulate this independent shift of 5 catheters for every patient. In theory, 59,049 simulations are required to mimic individual catheter shift for every Contura ${ }^{\circledR}$ MLB patient because each catheter has possible 9 simulations independently such as 8 shift cases ranging from $-4 \mathrm{~mm}$ to $+4 \mathrm{~mm}$ and a case without shift. Instead, in this study, 8 simulations were performed for each Contura ${ }^{\circledR}$ MLB patient the same as for MammoSite ${ }^{\circledR}$ patients to investigate the maximum range of dose variation for each simulation.

Therefore, a total of 392 plans (8 simulations for 49 patients) was retrospectively produced and compared to clinical treatment plans. For each simulated plan, seven dosimetric parameters were evaluated as follow: PTV_EVAL $\mathrm{D}_{95}, \mathrm{~V}_{100}$, and $\mathrm{V}_{90}$ values for target dosimetry; skin and rib $D_{\max }$ values, and normal breast tissue $V_{150}$ and $V_{200}$ values for OARs dosimetry.

\section{Results}

For each patient, eight simulated plans were generated and seven dosimetric parameters for each simulated plan were compared with those of treatment plan (reference plan). Hence, the total 2744 dosimetric data points from 392 retrospectively simulated plans were compared to the 343 reference dosimetric data points from 49 clinical treatment plans. Table 1 classifies dosimetric changes into three categories: increase, decrease, and invariance in comparison with dosimetry data in the reference treatment plan. PTV_EVAL dosimetric indices $\left(\mathrm{D}_{95}, \mathrm{~V}_{100}\right.$, and $\mathrm{V}_{90}$ ) were decreased in most simulations $(96.4 \%)$ while increased in $3.6 \%$ simulations. Skin and rib $\mathrm{D}_{\max }$ values were increased in 51\% simulations, decreased in $48.1 \%$ simulations, and invariant in $0.9 \%$ simulations. For normal breast tissue dosimetry, $\mathrm{V}_{150}$ value was increased in $58.2 \%$ simulations, decreased in $32.4 \%$ simulations, and invariant in $9.4 \%$ simulations. $\mathrm{V}_{200}$ value was increased in most simulations $(92.1 \%$ ) while decreased in $6.6 \%$ simulations. It was invariant for $1.3 \%$ simulations.
Table 1. Number of cases for dosimetric changes (3 categories) due to positioning uncertainty simulation (up to $\pm 4 \mathrm{~mm}$ shift from the reference source positions). The total number of simulated plans is 392 for 49 patients (8 simulations per patient)

\begin{tabular}{|c|c|c|c|}
\hline Dosimetric indices & Decrease & Invariance & Increase \\
\hline PTV_EVAL D $95[\%]$ & 382 & 0 & 10 \\
\hline PTV_EVAL V $100[\%]$ & 380 & 0 & 12 \\
\hline PTV_EVAL V $90[\%]$ & 372 & 0 & 20 \\
\hline Skin $D_{\max }[\%]$ & 168 & 2 & 222 \\
\hline $\operatorname{Rib} D_{\max }[\%]$ & 209 & 5 & 178 \\
\hline $\begin{array}{l}\text { Normal breast tissue } \\
V_{150}[c c]\end{array}$ & 127 & 37 & 228 \\
\hline $\begin{array}{l}\text { Normal breast tissue } \\
V_{200}[c c]\end{array}$ & 26 & 5 & 361 \\
\hline \multicolumn{4}{|c|}{$\begin{array}{l}D_{95}[\%]-\text { dose to the } 95 \% \text { volume of interest relative to the prescribed dose, } \\
V_{100}[\%] \text { and } V_{90}[\%] \text { - relative percentage of volume of interest, which receives } \\
100 \% \text { and } 90 \% \text { of the prescribed dose, respectively; } D_{\max }[\%] \text { - maximal point } \\
\text { dose of volume of interest relative to the prescribed dose; } V_{150}[\mathrm{cc}] \text { and } V_{200}[\mathrm{cc}] \\
\text { - absolute volume of interest, which receives } 150 \% \text { and } 200 \% \text { of the prescribed } \\
\text { dose, respectively }\end{array}$} \\
\hline
\end{tabular}

When it comes to presenting group data, if most data in a group show a trend for change (i.e., decrease), descriptive statistics of the group is useful to show the trend. Hence, statistical box graphs were used to show a trend of deviation of PTV_EVAL $D_{95}, \mathrm{~V}_{100}$, and $\mathrm{V}_{90}$ (Figure 1), and normal breast tissue $V_{200}$ values (Figure 2). On contrast, if each individual datum is case-specific within the group, the variation of individual datum cannot be shown with group statistics. Positive and negative deviations can be cancelled each other when grouping the individual data. Therefore, individual line graphs were employed to show individual variations of skin and rib $\mathrm{D}_{\max }$ (Figure 3). To avoid the cancellation between positive and negative changes within a simulation group, particularly for skin and rib $D_{\max }$ values and normal breast tissue $V_{150}$ values, absolute variation (modulus of change: $\mid(\Delta$ Value $) \mid$ $=\mid\left(\right.$ Value $_{\text {Simulation }}-$ Value $\left.\left._{\text {Reference }}\right) \mid\right)$ was also investigated between 8 simulation groups.

\section{Clinical treatment plan (reference plan)}

The Contura ${ }^{\circledR}$ registry protocol target coverage requirement (PTV_EVAL $D_{95} \geq 95 \%$ ) was satisfied for 44 clinical treatment plans while violated for one single lumen MammoSite ${ }^{\circledR}$ plan and four Contura ${ }^{\circledR}$ plans. In those five cases, PTV_EVAL coverage was compromised to reduce rib $D_{\max }$ because the balloon was located proximally to the rib $(<5 \mathrm{~mm})$. The average and maximum rib $\mathrm{D}_{\max }$ value for those five cases was $150.5 \%$ and $160.9 \%$ of the prescribed dose, respectively. Furthermore, two out of those five patients had the Contura ${ }^{\circledR}$ balloon proximal to skin as well. Hence, treatment plan was optimized to reduce skin $D_{\max }$ to $\leq 125 \%$ of the prescribed dose $(105 \%$ and $113 \%$ of the prescribed dose for these two Contura ${ }^{\circledR}$ MLB patients). For those five cases, the minimum target coverage requirement $\left(\mathrm{V}_{90}>90\right)$ was satisfied and the 
A

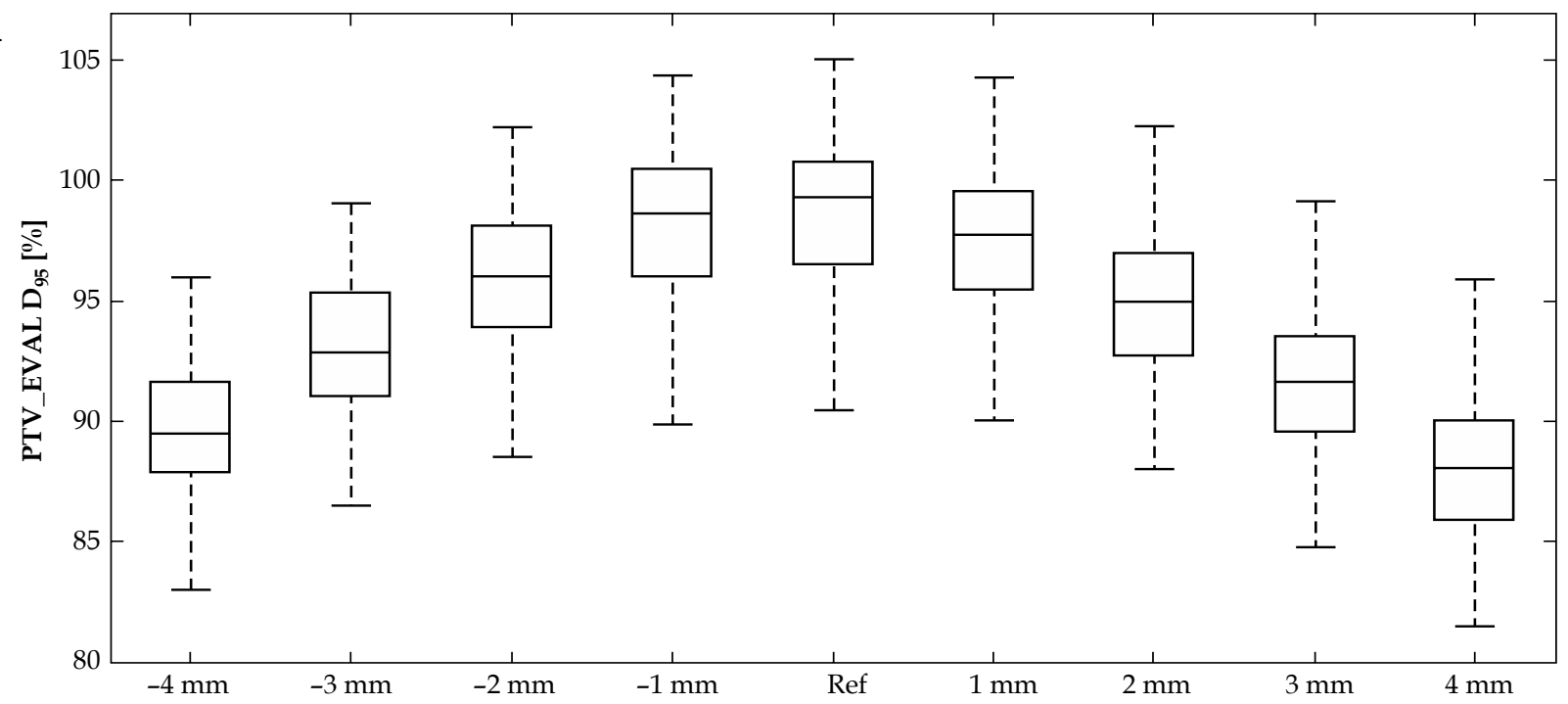

B

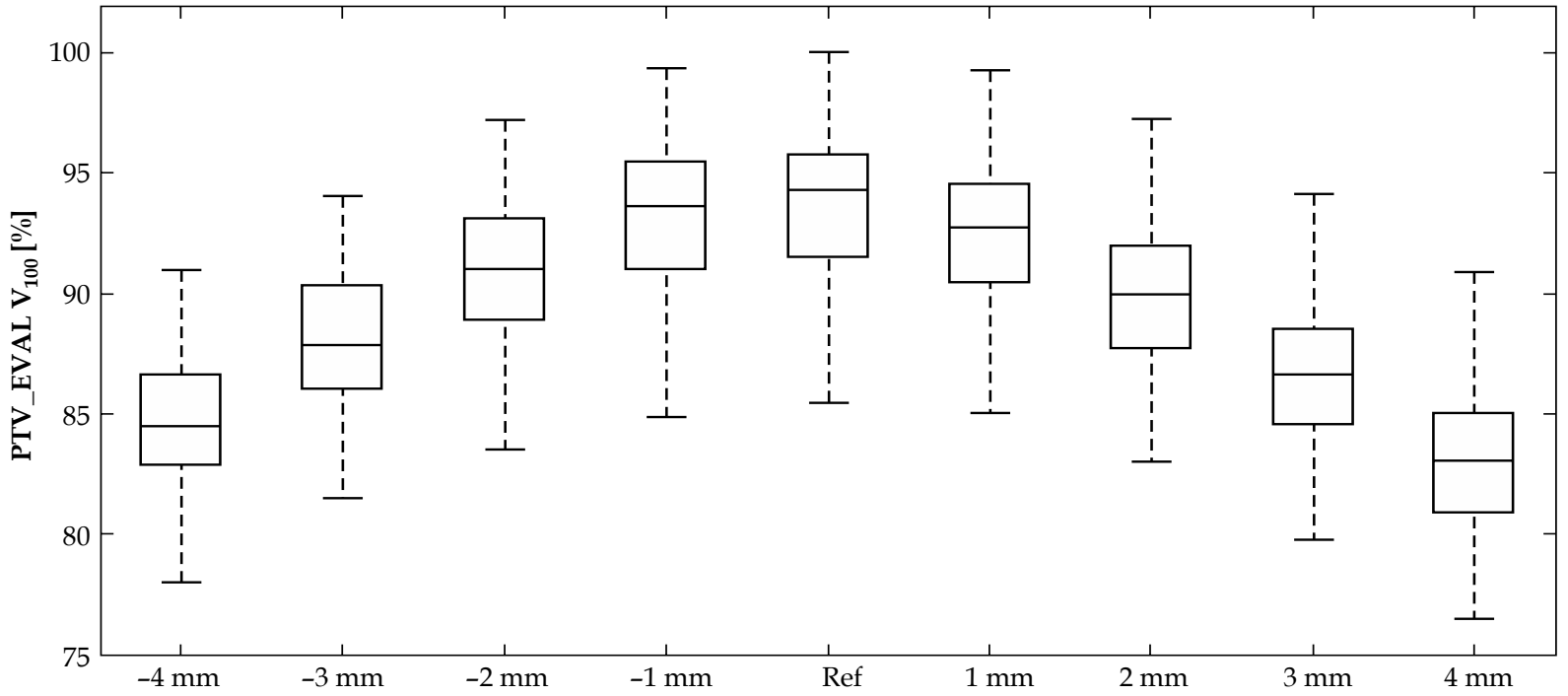

C

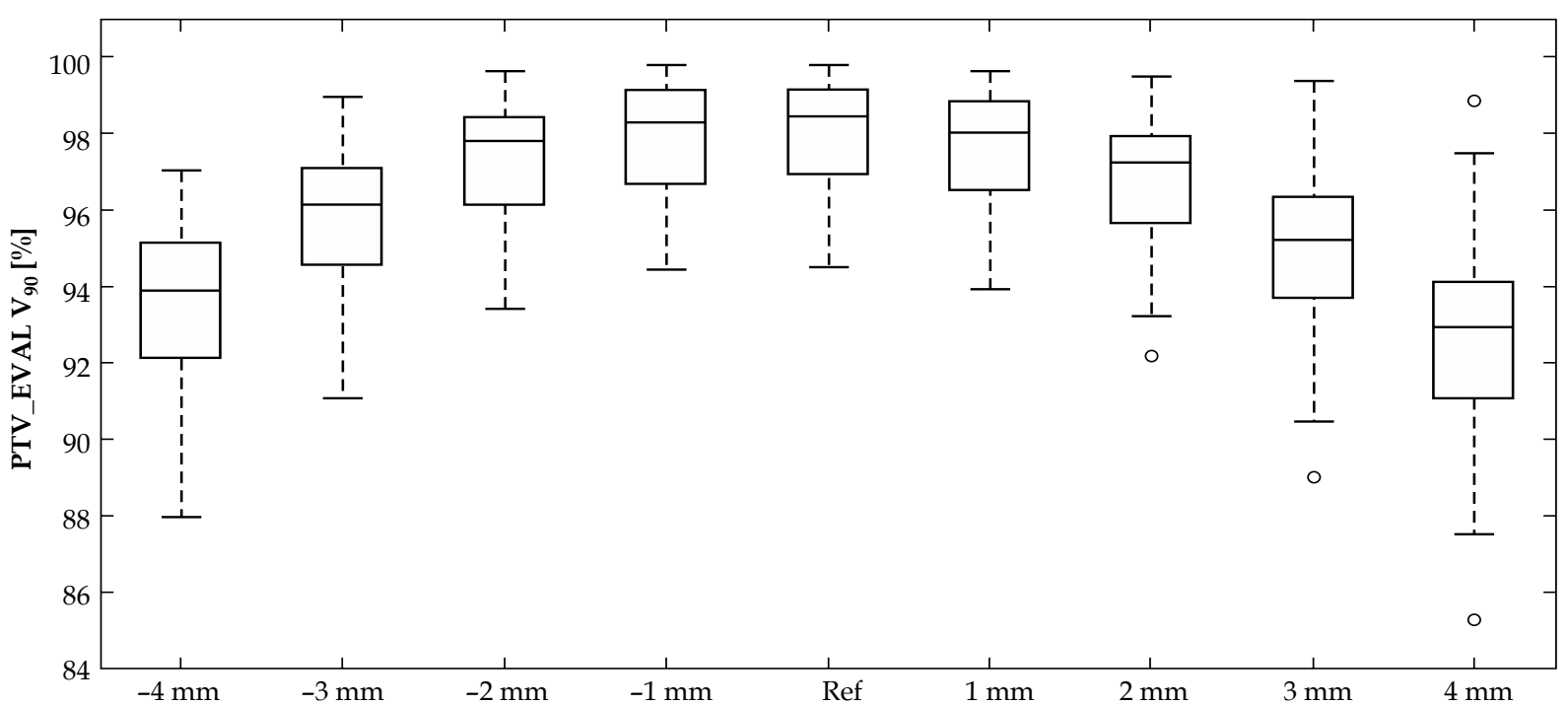

Fig. 1. PTV_EVAL dosimetric changes due to source-positioning shift ranging from $-4 \mathrm{~mm}$ to $+4 \mathrm{~mm}$ for (A) $\mathrm{D}_{95}$, (B) $\mathrm{V}_{100}$, and (C) $V_{90}$ values. Nine statistical box graphs show eight simulations as well as the reference (Ref) data. Each statistical box graph summarizes 49 data points and each parallel bar in each box graph represents $25^{\text {th }}, 50^{\text {th }}$, and $75^{\text {th }}$ percentile values. Two whiskers represent $99.3 \%$ coverage $\left(0.35^{\text {th }}-99.65^{\text {th }}\right.$ percentile) of data assuming that the data follow normal distribution. The open circles show outliers for each group 


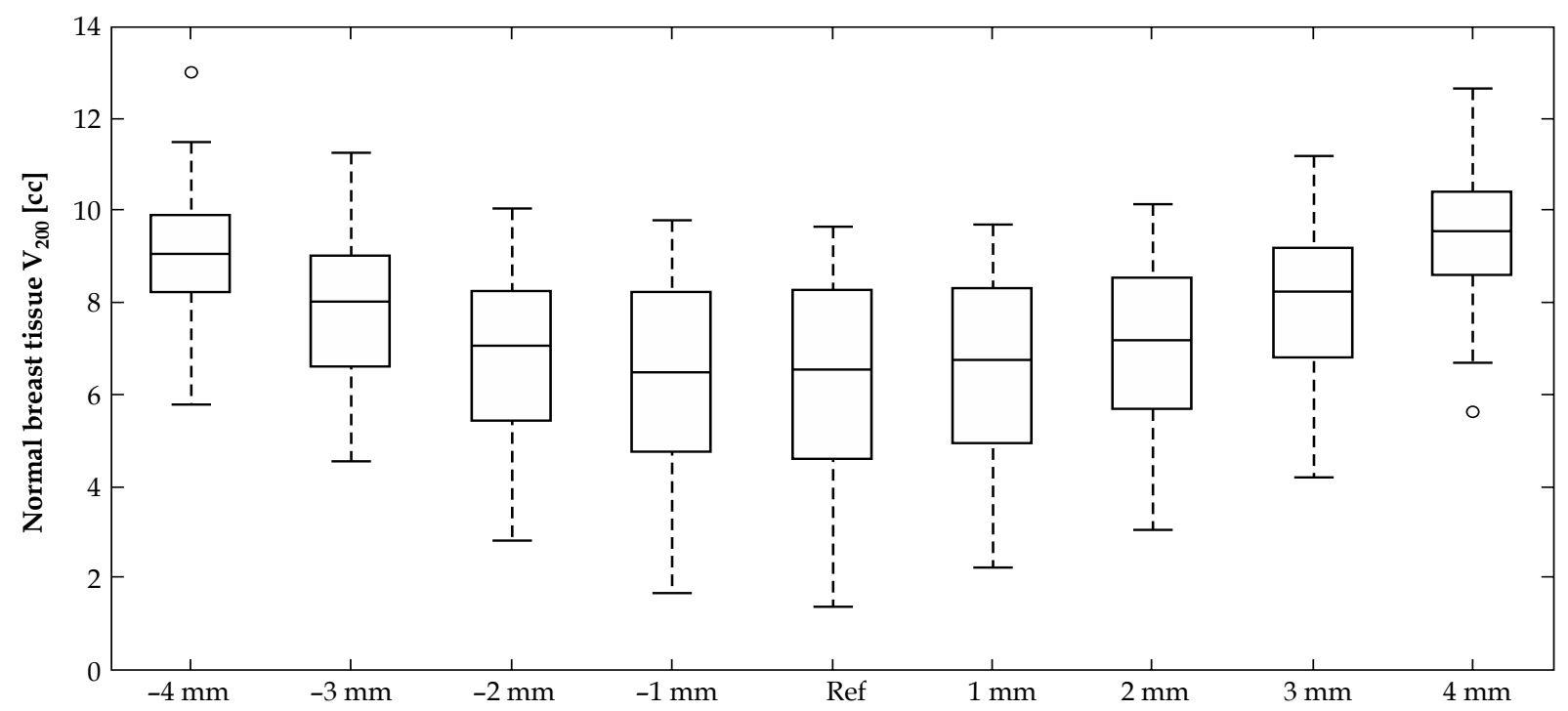

Fig. 2. Variation of normal breast tissue $V_{200}$ value due to source-positioning shift ranging from $-4 \mathrm{~mm}$ to $+4 \mathrm{~mm}$ for 49 patients.

Nine statistical box graphs represent group data for eight simulations as well as reference data

lowest PTV_EVAL V 90 value was $94.9 \%$ of the PTV_EVAL volume.

Skin $D_{\max }$ was $\leq 125 \%$ of the prescribed dose for 47 patients while $>125 \%$ for two Contura ${ }^{\circledR}$ patients. One patient $\left(137.5 \%\right.$ of skin $\left.\mathrm{D}_{\max }\right)$ had a stringent dose constraint to normal breast tissue $V_{200}$ [cc] (9.7 cc), and the other patient $\left(129.4 \%\right.$ of skin $\left.D_{\max }\right)$ had a higher priority to target coverage than skin $\mathrm{D}_{\max }$ in volume optimization. Rib $D_{\max }$ was $\leq 145 \%$ of the prescribed dose for 44 patients while $>145 \%$ for two single-lumen MammoSite ${ }^{\circledR}$ patients and three Contura ${ }^{\circledR}$ patients. In the treatment planning for single lumen MammoSite ${ }^{\circledR}$ patients, rib structure was not considered as an OAR. For three Contura ${ }^{\circledR}$ plans, PTV_EVAL coverage $\left(D_{95} \geq 95 \%\right)$ constraint had a higher priority than rib $D_{\max }$ and the highest value of rib $D_{\max }$ was $156 \%$ of the prescribed dose. Normal breast tissue $\mathrm{V}_{150}$ [cc] and $\mathrm{V}_{200}$ [cc] values were always $<50 \mathrm{cc}$ (maximum of $44.5 \mathrm{cc}$ ) and < $10 \mathrm{cc}$ (maximum of $9.7 \mathrm{cc}$ ), respectively. The descriptive statistics for clinical treatment plans was summarized in Table 2 as a reference (Ref) group. Four descriptive statistical parameters were used to summarize group data: average (Mean), standard deviation (SD), minimum (Min), and maximum (Max) values.

\section{Target coverage $\left(P T V_{-} E V A L D_{95}, V_{100}\right.$ and $\left.V_{90}\right)$ variation}

Due to positioning uncertainty, PTV_EVAL dosimetric data were decreased in majority of simulations $(96.4 \%)$ although increased in 42 out of 1176 simulations (Table 1). In those elevated simulation cases, $93 \%$ (39 out of 42 ) was $\pm 1 \mathrm{~mm}$ shift scenario and only 3 simulation cases were $\pm 2 \mathrm{~mm}$ shift scenarios. The magnitude of PTV_EVAL dosimetric increase was small: average (maximum) of $0.2 \%(0.4 \%)$ for $\mathrm{D}_{95}$ value; $0.2 \%(0.5 \%)$ for $\mathrm{V}_{100}$ value; $0.1 \%(0.3 \%)$ for $\mathrm{V}_{90}$ value.

Consistently, the larger source position uncertainty resulted in the larger reduction of PTV_EVAL coverage as shown in Figure 1. Compared to data of the reference plan, average reduction of PTV_EVAL dosimetrics was gradually increased as magnitude of shift was increased: from $1.0 \%( \pm 1 \mathrm{~mm}), 3.3 \%$ ( $\pm 2 \mathrm{~mm}), 6.3 \%( \pm 3 \mathrm{~mm})$ to $9.8 \%( \pm 4 \mathrm{~mm})$ for $\mathrm{D}_{95}$ value; from $1.0 \%( \pm 1 \mathrm{~mm}), 3.1 \%$ $( \pm 2 \mathrm{~mm}), 5.8 \%( \pm 3 \mathrm{~mm})$ to $8.7 \%( \pm 4 \mathrm{~mm})$ for $\mathrm{V}_{100}$ value; from $0.2 \%$ ( $\pm 1 \mathrm{~mm}), 1.0 \%$ ( $\pm 2 \mathrm{~mm}), 2.7 \%( \pm 3 \mathrm{~mm})$ to $5.1 \%( \pm 4 \mathrm{~mm})$ for $\mathrm{V}_{90}$ value. The $\mathrm{V}_{90}$ value (Figure 1C) was less susceptible to the positioning uncertainty than $\mathrm{D}_{95}$ (Figure $1 \mathrm{~A}$ ) and $\mathrm{V}_{100}$ (Figure $1 \mathrm{~B}$ ) values because $\mathrm{V}_{90}$ value of reference plans was already close to its upper limit $(100 \%)$ with average of $98 \%$ and standard deviation of $1.4 \%$ (Table 1). A nonparametric repeated measures ANOVA (analysis of variance), Friedman test, was performed for 8 different simulation groups as well as the reference group and showed statistically significant difference among groups ( $p$ value $<0.0001$ ).

The positioning uncertainty of $\geq \pm 3 \mathrm{~mm}$ decreased average $\mathrm{D}_{95}$ value from $98.7 \%$ (Ref) to lower than the Contura $^{\circledR}$ registry dosimetric goal $(\geq 95 \%$ of the prescribed dose), except for $+2 \mathrm{~mm}$ shift (94.9\%). In addition, average $\mathrm{V}_{100}$ value of $92.4 \%$ (reference plan) was reduced to $<90 \%$ due to as small as $\pm 2 \mathrm{~mm}$ shift $(89.9 \%$ for $-2 \mathrm{~mm}$ shift and $88.9 \%$ for $+2 \mathrm{~mm}$ shift). Furthermore, the minimal target coverage requirement $\left(\mathrm{V}_{90} \% \geq 90 \%\right)$ was violated for 13 large positioning uncertainty scenarios ( 1 for $+3 \mathrm{~mm}$ shift and 12 for $\pm 4 \mathrm{~mm}$ shift).

\section{OARs dose variation}

\section{Skin and rib $D_{\max }$ variation}

To display skin and rib $\mathrm{D}_{\max }$ variation for 8 simulation scenarios, the 49 patients data were ranked based on the value of the reference plans and categorized into two groups: low dose group (25 patients) and high dose group (24 patients). Figure 3 shows skin $D_{\max }$ variation (A) and rib $D_{\max }$ variation (B), respectively. In each Figure, low dose group is presented in the left panel and high dose group in the right panel. The variation of skin and rib $\mathrm{D}_{\max }$ values was highly case-specific. It was in- 

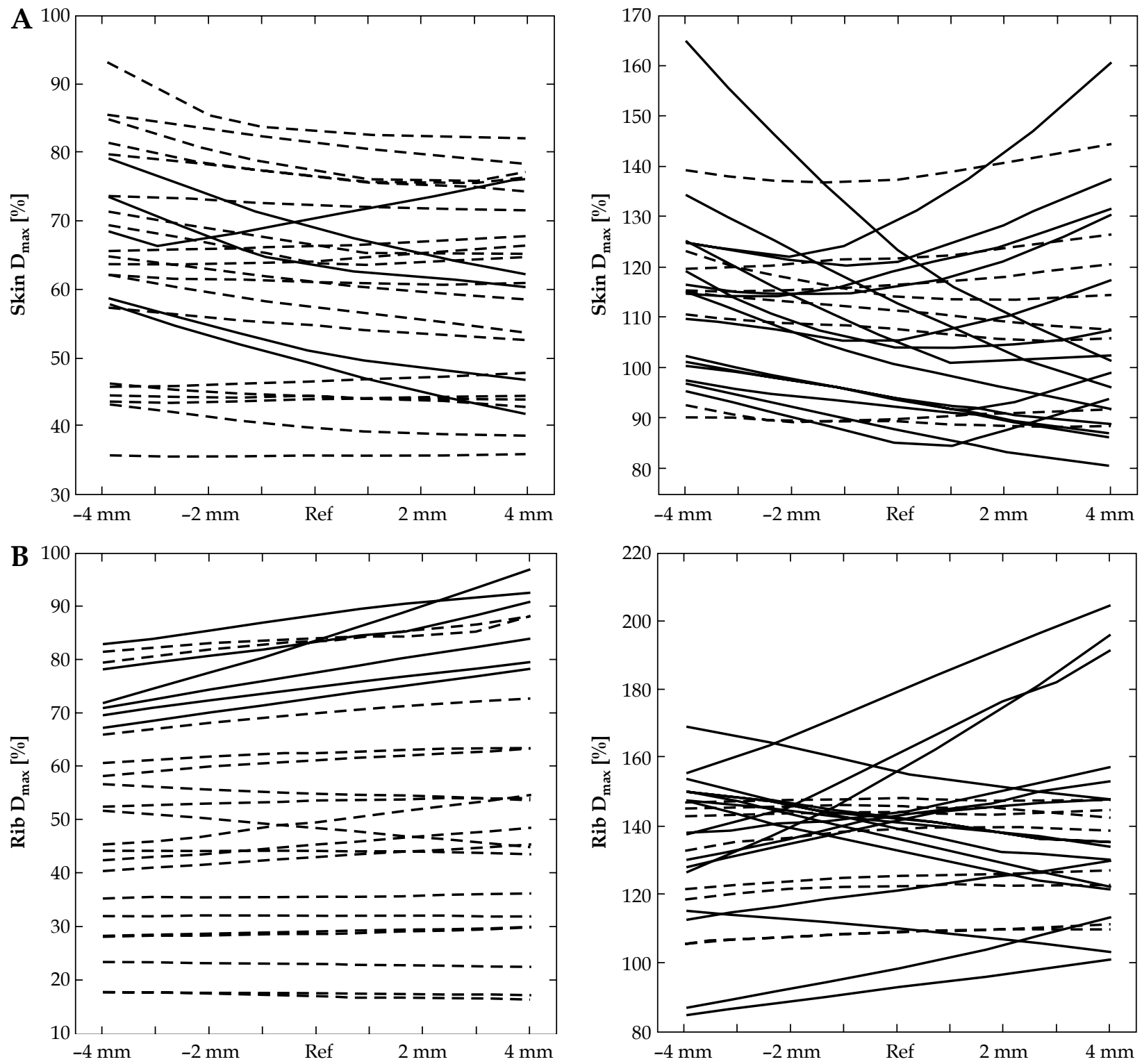

Fig. 3. Skin $D_{\max }$ (A) and rib $D_{\max }$ (B) variation due to positioning uncertainty ranging from $-4 \mathrm{~mm}$ to $+4 \mathrm{~mm}$. The 49 data in the reference plans were ranked and categorized into low dose (25 data) and high dose (24 data) groups. The individual line graph shows each patient data points for low dose group (left panel) and high dose group (right panel), respectively. For each patient, solid line is used if the average modulus of change $(\mid \Delta$ Value $\mid)$ is $>3 \%$ while dash line is used in case of $\mid \Delta$ Value $\mid \leq 3 \%$

Table 2. Descriptive statistics of dosimetric parameters for the reference group. Refer to footnote in Table 1 for dosimetric notation of $\mathrm{D}_{95}[\%], \mathrm{V}_{100}[\%], \mathrm{V}_{90}[\%], \mathrm{D}_{\max }[\%], \mathrm{V}_{150}[\mathrm{cc}]$, and $\mathrm{V}_{200}[\mathrm{cc}]$

\begin{tabular}{lcccc} 
Dosimetric indices & Mean & SD & Min & Max \\
\hline PTV_EVAL $D_{95}[\%]$ & 98.7 & 3.1 & 90.5 & 105.1 \\
\hline PTV_EVAL $V_{100}[\%]$ & 92.4 & 3.3 & 83.7 & 97.4 \\
\hline PTV_EVAL $V_{90}[\%]$ & 98.0 & 1.4 & 94.5 & 99.8 \\
\hline Skin $D_{\max }[\%]$ & 83.4 & 27.3 & 35.5 & 137.5 \\
\hline Rib $D_{\max }[\%]$ & 93.7 & 45.9 & 16.9 & 179.0 \\
\hline Normal breast tissue $\mathrm{V}_{150}[\mathrm{cc}]$ & 32.3 & 5.1 & 23.0 & 44.5 \\
\hline Normal breast tissue $\mathrm{V}_{200}[\mathrm{cc}]$ & 6.2 & 2.2 & 1.4 & 9.7
\end{tabular}

Mean - average value, SD - standard deviation, Min - minimal value, Max - maximal value 
creased in 400 simulation cases while decreased in 377 simulation cases (Table 1). It was invariant in 7 simulation cases. In some patients, skin and rib $\mathrm{D}_{\max }$ change was large. For instance, for a certain patient, $-1 \mathrm{~mm}$ and $-4 \mathrm{~mm}$ shifts increased skin $\mathrm{D}_{\max }$ by $9.1 \%$ and $41.9 \%$ of the prescribed dose, respectively. For another patient, $+1 \mathrm{~mm}$ and $+4 \mathrm{~mm}$ shift increased rib $\mathrm{D}_{\max }$ by $9.3 \%$ and $40.2 \%$ of the prescribed dose, respectively. In contrast, skin and rib $\mathrm{D}_{\max }$ change was small in some patients. For example, for a patient $-4 \mathrm{~mm}$ shift reduced skin $\mathrm{D}_{\max }$ by only $0.03 \%$ of the prescribed dose and $+4 \mathrm{~mm}$ shift reduced rib $\mathrm{D}_{\max }$ by only $0.14 \%$ of the prescribed dose for another patient. Therefore, skin and rib $\mathrm{D}_{\max }$ variation is highly case-specific and independent of direction and magnitude of shift.

However, the modulus of the change $\left(\mid \Delta\left(\right.\right.$ Skin $\left.D_{\max }\right) \mid$ and $\mid \Delta\left(\right.$ Rib $\left.\left.D_{\max }\right) \mid\right)$ showed statistically significant difference between 8 different simulation groups ( $p$ value $<0.0001$ ) using a nonparametric repeated measures ANOVA, Friedman test. Hence, in general, the larger source position uncertainty can cause the larger $\mid \Delta$ (Skin $\left.\mathrm{D}_{\max }\right) \mid$ and $\left|\Delta\left(\mathrm{Rib} \mathrm{D}_{\max }\right)\right|$. In addition, for each simulation, statistical comparison was performed between $\mid \Delta\left(\right.$ Skin $\left.D_{\max }\right) \mid$ and $\mid \Delta\left(\right.$ Rib $\left.D_{\max }\right) \mid$ using nonparametric test (Mann-Whitney Test). All $8 p$ values corresponding to each simulation were $>0.75$ and there was no statistical difference of $\left|\Delta\left(D_{\max }\right)\right|$ between skin and rib $D_{\max }$ values.

Furthermore, to investigate the significance of dose variation difference between low dose ( 25 patients) and high dose (24 patients) groups, $\mid \Delta$ (Value)| for 8 simulations were averaged for each patient and statistical analysis was performed between two patient groups. A non-parametric test (Mann-Whitney) was used because the sample size was not sufficiently large and statistics of data did not follow normal distribution. Although the individual dose variation was highly case-specific as seen in Figure 3, statistical analysis (Table 3) demonstrated that high dose group showed higher average $\left|\Delta\left(D_{\text {max }}\right)\right|$ than low dose group for skin $D_{\text {max }}(p=0.0003)$ and rib $\mathrm{D}_{\max }(p=0.0004)$. For the combined skin and rib $\mathrm{D}_{\max }$ data, statistical difference was more pronounced $(p<0.0001)$ between low dose and high dose groups.

\section{Normal breast tissue dose $\left(V_{150}\right.$ and $\left.V_{200}\right)$ variation}

The normal breast tissue $V_{150}$ value change was casespecific: either increased (58\%) or decreased $(32 \%)$ in Table 1. It was invariant for $10 \%$ of simulation cases. The variation of $\mathrm{V}_{150}$ value was too small to be noticeable for all patients. For two largest simulations ( $\pm 4 \mathrm{~mm}$ shift), the average $\left|\Delta\left(V_{150}\right)\right|$ was $0.2 \mathrm{cc}$. Even, in the worst scenario, the maximum deviation of $\mathrm{V}_{150}$ value was less than $2 \mathrm{cc}$ : $1.6 \mathrm{cc}$ and $0.8 \mathrm{cc}$ for $-4 \mathrm{~mm}$ and $+4 \mathrm{~mm}$ shift scenarios, respectively.

The normal breast tissue $\mathrm{V}_{200}$ value was increased in majority of simulations (92\%, Table 1). The amount of elevation was gradually increased as the magnitude of positioning uncertainty was increased as shown in Figure 2. The average $V_{200}$ value was $6.2 \mathrm{cc}$ at the reference plans and increased from $6.4 \mathrm{cc}( \pm 1 \mathrm{~mm}), 7.0 \mathrm{cc}( \pm 2 \mathrm{~mm}), 8.0 \mathrm{cc}$ $( \pm 3 \mathrm{~mm})$ to $9.2 \mathrm{cc}( \pm 4 \mathrm{~mm})$. The average increase of $V_{200}$ value was as high as up to $2.9 \mathrm{cc}$ and $3.2 \mathrm{cc}$ for $-4 \mathrm{~mm}$ and $+4 \mathrm{~mm}$ shift scenarios, respectively. The worst case of $\geq \pm 2 \mathrm{~mm}$ simulations violated the requirement of $\mathrm{V}_{200}$ $(>10 \mathrm{cc})$. The Friedman test performed among 8 different simulation groups and the reference group and it showed statistically significant difference among groups ( $p$ value $<0.0001)$.

\section{Discussion}

Due to the source positioning uncertainty, the prescribed dose isodose curve which originally conforms to the outer surface of the spherical shell will be shifted along the axis of balloon either proximally or distally depending upon the direction and magnitude of positioning inaccuracy. Hence, PTV_EVAL coverage $\left(D_{95}, V_{100}\right.$ and $V_{90}$ in Figure 1) deterioates due to any shift of the optimal dose distribution along the axis of balloon. However, in some clinical cases, the position of total treatment length (sequential sum of possible dwell positions) inside the balloon was slightly off from the center of balloon. In this study, the source stepping size was set to $5 \mathrm{~mm}$ the same as the physical size of ${ }^{192}$ Ir source. For those cases (42 out of 1176 cases in Table 1 ), small shift along the axis of balloon could increase target coverage (PTV_EVAL $\mathrm{D}_{95}, \mathrm{~V}_{100}$ or $\mathrm{V}_{90}$ values): 39 cases with $\pm 1 \mathrm{~mm}$ shift and 3 cases with \pm 2 mm shift.

Table 3. Statistical comparison between low dose and high dose groups of skin and rib $D_{\max }$ changes. For each patient, the modulus of $D_{\max }$ change was averaged for all 8 positioning uncertainty simulations. The 49 patients' data were ranked based on the $D_{\max }$ value of reference plan and categorized into two groups (low and high doses of $\left.D_{\max }\right)$

\begin{tabular}{|c|c|c|c|c|c|}
\hline & Group & Sample size & Mean & Standard Deviation & ${ }^{*} p$ value \\
\hline \multirow[t]{2}{*}{ Skin $D_{\max }$} & Low dose & 25 & 2.0 & 1.5 & 0.0003 \\
\hline & High dose & 24 & 5.3 & 4.3 & \\
\hline \multirow[t]{2}{*}{ Rib $D_{\max }$} & Low dose & 25 & 1.9 & 1.8 & 0.0004 \\
\hline & High dose & 24 & 6.2 & 5.6 & \\
\hline \multirow{2}{*}{$\begin{array}{l}\text { Combined data } \\
\text { (Skin/Rib } D_{\max } \text { ) }\end{array}$} & Low dose & 49 & 1.9 & 0.2 & $<0.0001$ \\
\hline & High dose & 49 & 5.7 & 4.9 & \\
\hline
\end{tabular}

"p value was computed using non-parametric test (Mann-Whitney test) 
In general, the $200 \%$ isodose line overlaps with the balloon surface and the normal breast tissue volume $\left(\mathrm{V}_{200}\right)$ receiving $200 \%$ of the prescribed dose is limted to $\leq 10 \mathrm{cc}$. If the $200 \%$ isodose line is spherical shape, it should always increase due to the shift of source positions. However, in clincially difficult case where skin and rib structures are proximally located to the balloon simultaneously, the optimal dose distribution should conform to the shape of outer surface of PTV_EVAL. The PTV_EVAL shape is not a spherical shell by excluding both spherical caps in skin and rib sides, resulting in ellipsoidal shape of $200 \%$ isodose curves. In addition, the asymmetry of total treatment length inside the balloon can induce the asymmtery of $200 \%$ isodose lines from the center of balloon. Because of these effects, normal breast tissue $V_{200}$ value can be decreased due to source-position uncertainty for some simulation cases ( 26 out of 392 cases in Table 1).

Schematic diagrams in Figure 4 depict three representative clinical cases for single lumen MammoSite ${ }^{\circledR}$ balloon applicator depending upon the minimal distances from the balloon to the skin/rib structures (skin spacing and rib spacing): (A) both spacings $\geq 0.7 \mathrm{~cm}$; (B) either spacing (particularly, skin spacing in this example) $<0.7 \mathrm{~cm}$; (C) both spacings $<0.7 \mathrm{~cm}$. In Case (A), an optimal dose distribution can be obtained with eight available dwell positions, comforming to the outer surface of spherical shell. Skin and rib $D_{\max }$ values are less than the prescribed dose because both spacings are $>1 \mathrm{~cm}$. In Case (B), skin $\mathrm{D}_{\text {max }}$ is more than the prescribed dose due to skin spacing of $<0.7 \mathrm{~cm}$. It can be reduced to less than the prescribed dose if MLB applicator is used. In Case (C), both skin and rib $\mathrm{D}_{\max }$ would be higher than the prescribed dose due to $<0.7 \mathrm{~cm}$ of skin and rib spacings. Both $\mathrm{D}_{\max }$ values can be reduced using MLB applicator and dose distribution may be ellipsoidal shape, conforming to the outer surface of PTV_EVAL (grey color in Figure 4C). However, even though MLB is able to reduce OARs dose with multi-lumen, the dose shaping capability is highly limited if the orientation of balloon insertion is vertical to the OARs: Orient $(\mathrm{V})$ in Figure $4 \mathrm{~B}$ and $4 \mathrm{C}$. All outer lumens are perpendicular to skin and rib and the minimal distances from each outer lumen to OARs is the same. Therefore, the best orientation of balloon insertion is parallel to the skin and rib: Orient $(\mathrm{P})$ in Figure 4 in order to maximize the dose shaping capability of MLB applicator.

The deviation of skin and rib $\mathrm{D}_{\max }$ values due to shift of source positions is highly dependent upon the relative location of skin and rib to the balloon. In brachytherapy, the inverse square law is the dominant factor to determine dose. The farther away from the balloon, the smaller skin or rib $\mathrm{D}_{\max }$ values, resulting in the smaller in their dose
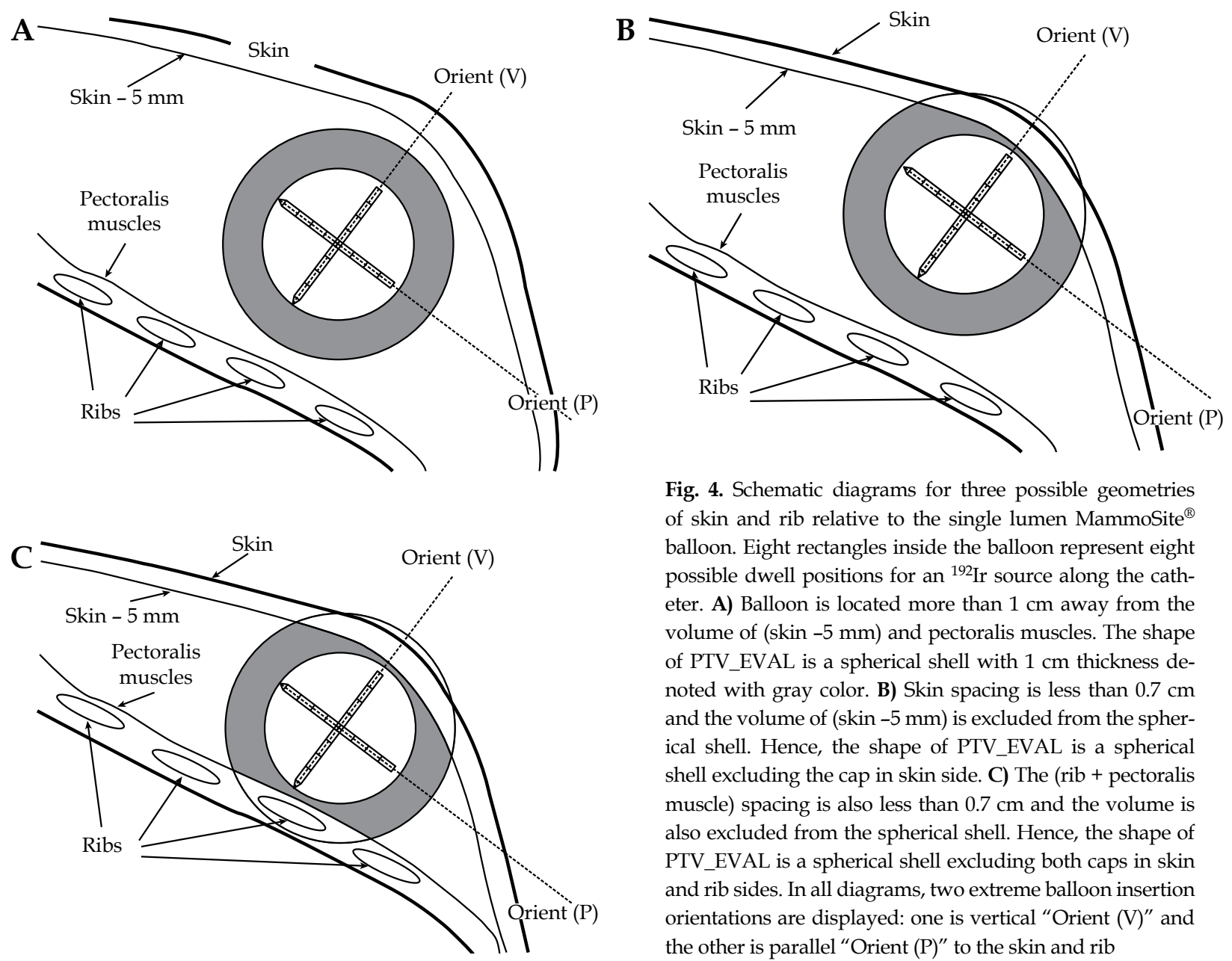

Fig. 4. Schematic diagrams for three possible geometries of skin and rib relative to the single lumen MammoSite ${ }^{\circledR}$ balloon. Eight rectangles inside the balloon represent eight possible dwell positions for an ${ }^{192}$ Ir source along the catheter. A) Balloon is located more than $1 \mathrm{~cm}$ away from the volume of (skin $-5 \mathrm{~mm}$ ) and pectoralis muscles. The shape of PTV_EVAL is a spherical shell with $1 \mathrm{~cm}$ thickness denoted with gray color. B) Skin spacing is less than $0.7 \mathrm{~cm}$ and the volume of (skin $-5 \mathrm{~mm}$ ) is excluded from the spherical shell. Hence, the shape of PTV_EVAL is a spherical shell excluding the cap in skin side. C) The (rib + pectoralis muscle) spacing is also less than $0.7 \mathrm{~cm}$ and the volume is also excluded from the spherical shell. Hence, the shape of PTV_EVAL is a spherical shell excluding both caps in skin and rib sides. In all diagrams, two extreme balloon insertion orientations are displayed: one is vertical "Orient (V)" and the other is parallel "Orient $(\mathrm{P})$ " to the skin and rib 
variation due to position uncertainty. In this study, another important factor affecting dose variation is the orientation of balloon insertion because the source-positioning uncertainty occurs exclusviely along the catheter(s). Examining two extreme cases in Figure 4 will elluciate the importance of the orientation of balloon insertion regarding the dose variation due to source-positioning uncertainty. If the orientation of balloon insertion is vertical to the skin/rib such as Orient (V) in Figure 4, the optimal dose distribution conformal to the outer surface of PTV_EVAL volume will be shifted either toward or away from skin/rib structures due to source positioning uncertainty. Hence, minimum distances from skin and rib structures to source positions change drastically due to the position uncertainty, thereby resulting in high dose variation of skin or rib $D_{\max }$ values. In contrast, if the orientation of balloon insertion is parallel to the skin/rib such as Orient $(\mathrm{P})$ in Figure 4, the shift of optimal dose distribution along the catheter will insignificantly affect skin and rib $\mathrm{D}_{\max }$ values because both structures are located relatively parallel to the balloon catheter. Therefore, the variation of minimum distances from skin and rib structures to source positions is small and thus the variation of skin/rib $\mathrm{D}_{\max }$ is also expected to be small.

Typically, low dose group has smaller dose variation of skin and rib $\mathrm{D}_{\max }$ than high dose group $(p<0.0001$ in Table 3) due to the inverse square law of brachytherapy as seen in Figure 3 (left panel vs. right panel). However, the effect of orientation of balloon insertion is noticeable in some patients in Figure 3. The average $\mid \Delta\left(\right.$ Skin $\left.D_{\text {max }}\right) \mid$ was more than $3 \%$ of the prescribed dose for 5 patients ( 5 solid lines in left panel of Figure 3A) in low dose group, while average $\mid \Delta\left(\right.$ Skin $\left.D_{\max }\right) \mid$ was less than $3 \%$ of the prescribed dose for 8 patients ( 8 dash lines in right panel of Figure 3A) in high dose group. The same phenomenon was observed for rib $\mathrm{D}_{\max }$ variation in Figure 3B. The average $\left|\Delta\left(\operatorname{Rib} D_{\max }\right)\right|$ was $>3 \%$ of the prescribed dose for 6 patients (6 solid lines in left panel of Figure 3B) in low dose group, while average $\left|\Delta\left(\mathrm{Rib} \mathrm{D}_{\max }\right)\right|$ was less than $3 \%$ of the prescribed dose for 8 patients $(8$ dash lines in right panel of Figure 3B) in high dose group.

For MLB applicator, the real clinical dosimetric variations due to the source positioning uncertainty should be less than the data from this study. Although in this study all five Contura ${ }^{\circledR}$ lumens were shifted all together, in the real clinical case the source positioning uncertainty occurs independently for each catheter. If the individual positioning uncertainty for each catheter happens in random fashion, the direction of shift simulation for each catheter can be opposite among multiple lumens and resultant dose perturbation is less than the data in this study. It is noted that the data in this study are limited to the extreme cases. However, still this study can provide clinically meaningful information such as a guideline on the range of maximum dose variation due to source positioning uncertainty in HDR balloon brachytherapy of breast cancer.

In general, HDR brachytherapy is performed in hypofractionation regimen and interfraction variation of the applicator shape and position relative to patient anatomy has become an issue in 3D image based HDR brachyther- apy. Kim et al. [12] measured interfraction change of MammoSite ${ }^{\circledR}$ balloon applicator and evaluated its dosimetric impact for 19 patients. They concluded that interfraction variations were patient-specific and fractionspecific. Though the average variation and its dosimetric impact are clinically insignificant, the maximum variation is not negligible and applicator shape and position should be verified prior to each fraction. In another study, Kim et al. [13] investigated the rotation issue of MLB applicator between fractions. Based on virtual simulation for device rotation in two representative clinical cases, it was reported that even device rotation as little as 30 degrees could negate the benefit from MLB applicator if the device rotation was disregarded. Hence, they concluded that verification and correction of device rotation is essential prior to delivery of each fraction. Recently, Kuo et al. [14] investigated geometric uncertainty and internal uncertainty for MLB applicator using 42 CT scans (one planning CT scan and 5 daily verification CT scans for 7 patients). They reported interfraction variation of balloon shape measurement in anterior-posterior and lateral directions, balloon volume, skin and rib spacing, and interfaction dosimetric variation for target coverage. In addition, each catheter was systematically shifted up to $\pm 4 \mathrm{~mm}$ the same as this study. The data were similar to this study. As magnitude of shift was increased, PTV_EVAL $\mathrm{V}_{90}$ was decreased from $0.5 \%( \pm 1 \mathrm{~mm}), 1.7 \%( \pm 2 \mathrm{~mm}), 3.5 \%$ $( \pm 3 \mathrm{~mm})$ to $5.7 \%( \pm 4 \mathrm{~mm})$. Hence, they came to the conclusion that $\pm 2 \mathrm{~mm}$ tolerance for HDR quality assurance is clinically reasonable although the maximum deviation should be avoided based on the verification of applicator prior to each fraction.

The American Brachytherapy Society (ABS) recently published consensus statement [15] for accelerated partial breast irradiation and provided clinical guidelines in order for clinicians to appropriately select patients. The dosimetry guidelines in this ABS consensus were referred to NSABP B-39/RTOG 0413 protocol for HDR balloon applicator and their update was recommended for new emerging applicators. Hence, dosimetry planning goals in this study followed the NSABP B-39/RTOG 0413 protocol for single lumen MammoSite ${ }^{\circledR}$ applicator and Contura $^{\circledR}$ registry protocol for Contura ${ }^{\circledR}$ applicator in accordance with the ABS consensus. Also, a guideline regarding clinical brachytherapy uncertainties was recently endorsed between Groupe Européen de Curiethérapie and the European Society for Therapeutic Radiology and Oncology (GEC-ESTRO) and AAPM, and it was recommended "to present data on the analyzed parameters and also their influence on absorbed dose for clinically-relevant dose parameters" [16]. This study followed the recommendations and source positioning uncertainty was simulated by an analyzed parameter (source position shift ranging from $\pm 1 \mathrm{~mm}$ to $\pm 4 \mathrm{~mm}$ ), and its impact was evaluated by clinically-relevant dosimetry changes in target coverage $\left(D_{95}, V_{100}\right.$, and $\left.V_{90}\right)$ and OAR dose (maximal point dose of skin and rib, and $\mathrm{V}_{150}$ and $\mathrm{V}_{200}$ values of normal breast tissue). Therefore, the data from this study can be used as general guidelines on dosimetric change due to catheter positioning uncertainty ranging from $\pm 1 \mathrm{~mm}$ to $\pm 4 \mathrm{~mm}$ for balloon HDR brachytherapy. In 
addition, special cases were emphasized where OARs are located close to the balloon and/or the orientation of balloon catheter is vertical to the OARs.

\section{Conclusions}

The tolerance of $\pm 2 \mathrm{~mm}$ recommended by AAPM TG 56 for catheter-positioning uncertainty is clinically acceptable in most clinical cases for HDR balloon brachytherapy of breast cancer. However, in a case where the dosimetry data of treatment plan are close to dosimetry limits of the clinical protocol, more caution should be paid because even $\pm 1 \mathrm{~mm}$ positioning uncertainty can make dosimetrics violate the clinical protocol dose limit. Particularly, special concern should be taken for the case where OARs are proximally located to the balloon and the orientation of balloon catheter is vertical to the OARs.

\section{Acknowledgments}

The author appreciates Dr. Mark G. Trombetta, MD (Allegheny General Hospital, Pittsburgh, Pennsylvania, USA), for providing clinical treatment plans and clinical research approval from the institutional review board in Allegheny General Hospital.

\section{Disclosure}

The author reports no conflict of interest.

\section{References}

1. Holt JG. AAPM Report No. 41: remote afterloading technology. Med Phys 1993; 20: 1761

2. Nath R, Anderson LL, Meli JA et al. Code of practice for brachytherapy physics: report of the AAPM Radiation Therapy Committee Task Group No. 56. American Association of Physicists in Medicine. Med Phys 1997; 24: 1557-1598.

3. Bensaleh S, Bezak E. Investigation of source position uncertainties \& balloon deformation in MammoSite brachytherapy on treatment effectiveness. Australas Phys Eng Sci Med 2010; 33: 35-44.

4. Bensaleh S, Bezak E. The impact of uncertainties associated with MammoSite brachytherapy on the dose distribution in the breast. J Appl Clin Med Phys 2011; 12: 3464.

5. Astrahan MA, Jozsef G, Streeter OE Jr. Optimization of MammoSite therapy. Int J Radiat Oncol Biol Phys 2004; 58: 220-232.

6. Dickler A, Kirk M, Choo J et al. Treatment volume and dose optimization of MammoSite breast brachytherapy applicator. Int J Radiat Oncol Biol Phys 2004; 59: 469-474.

7. Kirk M, Hsi WC, Dickler A et al. Surface optimization technique for MammoSite breast brachytherapy applicator. Int $J$ Radiat Oncol Biol Phys 2005; 62: 366-372.

8. Kim Y, Trombetta MG, Miften M. Comparison of single and multiple dwell position methods in MammoSite high dose rate (HDR) brachytherapy planning. J Appl Clin Med Phys 2010; 11: 3235.

9. NSABP B-39/RTOG 0413 protocol, “A randomized phase III study of conventional whole breast irradiation (WBI) versus partial breast irradiation (PBI) for women with stage 0 , I or II breast cancer"; available at http://rpc.mdanderson.org/ rpc/credentialing/files/B39_Protocol1.pdf

10. Kim Y, Trombetta MG. Objective method to report planner-independent skin/rib maximal dose in balloon-based high dose rate (HDR) brachytherapy for breast cancer. Med Phys 2011; 38: 2053-2057.

11. Arthur DW, Vicini FA, Todor DA et al. Contura Multi-Lumen Balloon breast brachytherapy catheter: comparative dosimetric findings of a phase 4 trial. Int J Radiat Oncol Biol Phys 2013; 86: 264-269.

12. Kim $Y$, Johnson M, Trombetta MG et al. Investigation of interfraction variations of MammoSite balloon applicator in high-dose-rate brachytherapy of partial breast irradiation. Int J Radiat Oncol Biol Phys 2008; 71: 305-313.

13. Kim Y, Trombetta MG. Dosimetric evaluation of multilumen intracavitary balloon applicator rotation in high-dose-rate brachytherapy for breast cancer. J Appl Clin Med Phys 2014; 15: 4429.

14. Kuo HC, Mehta KJ, Hong L et al. Day to day treatment variations of accelerated partial breast brachytherapy using a multi-lumen balloon. J Contemp Brachytherapy 2014; 6: 68-75.

15. Kirisits C, Rivard MJ, Baltas D et al. Review of clinical brachytherapy uncertainties: analysis guidelines of GEC-ESTRO and the AAPM. Radiother Oncol 2014; 110: 199-212.

16. Shah C, Vicini F, Wazer DE et al. The American Brachytherapy Society consensus statement for accelerated partial breast irradiation. Brachytherapy 2013; 12: 267-277. 